\title{
Genetic Diversity of Brucella Reference and Non-reference Phages and Its Impact on Brucella-Typing
}

\begin{abstract}
Jens A. Hammerl ${ }^{*}$, Cornelia Göllner ${ }^{1}$, Claudia Jäckel ${ }^{1}$, Holger C. Scholz ${ }^{2}$, Karsten Nöckler ${ }^{1}$, Jochen Reetz ${ }^{1}$, Sascha Al Dahouk ${ }^{1}$ and Stefan Hertwig ${ }^{1}$

${ }^{1}$ Department of Biological Safety, German Federal Institute for Risk Assessment, Berlin, Germany, ${ }^{2}$ German Center for Infection Research, Bundeswehr Institute of Microbiology, Munich, Germany
\end{abstract}

Virulent phages have been used for many years to type Brucella isolates, but until recently knowledge about the genetic makeup of these phages remains limited. In this work the host specificity and genomic sequences of the original set (deposited in 1960) of VLA Brucella reference phages Tb, Fi, Wb, Bk2, R/C, and Iz were analyzed and compared with hitherto described brucellaphages. VLA phages turned out to be different from homonymous phages in other laboratories. The host range of the phages was defined by performing plaque assays with a wide selection of Brucella strains. Propagation of the phages on different strains did not alter host specificity. Sequencing of the phages $\mathrm{Tb} v, \mathrm{Fi}_{\mathrm{v}}, \mathrm{Wb}$, and $\mathrm{R} / \mathrm{C}_{\mathrm{V}}$ revealed nucleotide variations when compared to same-named phages previously described by other laboratories. The phages Bk2 $\mathrm{V}$ and Iz $v$ were sequenced for the first time. While Bk2 $v$ exhibited the same deletions as $W b_{v}, I_{v}$ possesses the largest genome of all Brucella reference phages. The duplication of a 301 bp sequence in this phage and the large deletion in $B k 2 v, W b v$, and R/CV may be a result of recombination caused by repetitive sequences located in this DNA region. To identify new phages as potential candidates for lysotyping, the host range and Single Nucleotide Polymorphisms (SNPs) of 22 non-reference Brucella phages were determined. The phages showed lysis patterns different from those of the reference phages and thus represent novel valuable candidates in the typing set.

Keywords: Brucella, phage, genome, virulent, lysotyping

\section{INTRODUCTION}

Brucellae are highly infectious and facultative intracellular bacterial pathogens causing brucellosis, a frequent zoonosis with more than 500,000 human cases reported worldwide every year (de Figueiredo et al., 2015). Infections may lead to reproductive failure and abortion in animals and a feverish multiorgan disease in humans. Up to now, 12 species were allocated to the genus Brucella (Godfroid et al., 2013). Six of them, B. melitensis, B. abortus, B. suis, B. canis, B. ovis, and $B$. neotomae, recovered from goats, cattle, pigs, dogs, sheep, and desert rats, respectively, belong to the "classical" or "historical" Brucella species (http://www.bacterio.net/brucella.html). Later on, "novel" Brucella species were isolated from cetaceans (B. ceti) and pinnipeds (B. pinnipedialis; Foster et al., 2007), voles (B. microti; Scholz et al., 2008b), baboons (B. papionis; 
Whatmore et al., 2014), red foxes (B. vulpis; Scholz et al., 2016) and from a human breast implant infection (B. inopinata; Scholz et al., 2010). Recently, a novel Brucella spp. reservoir in amphibians (e.g., Big-eyed tree frog: Leptopelis verniculatus; African bullfrogs: Pyxicephalus edulis; White's tree frog: Litoria caerulea) was discovered (Eisenberg et al., 2012; Fischer et al., 2012; Whatmore et al., 2015; Soler-Lloréns et al., 2016). Genetically, all Brucella species are closely related exhibiting genome similarities of $>90 \%$ at the nucleotide level (Al Dahouk et al., 2010). Furthermore, because of the close genetic relationship of several genetic loci (e.g., 16S rRNA, 98.7\% and recA, $85.5 \%$ ) and a biochemical profile similar to Ochrobactrum spp., particularly atypical Brucella species like B. microti and $B$. inopinata are often misidentified using commercially available biochemical test systems (Scholz et al., 2008a,c).

Identification and subtyping of brucellae is time-consuming and laborious. Suspicious colonies on agar plates are primarily identified by a slide agglutination test using polyvalent Brucella antiserum (anti-S serum; Alton et al., 1975). Alternatively, PCR detection systems targeting the bcsp31 gene and the intergenic sequence IS711 exist that are suited for the molecular detection of Brucella spp. (Baily et al., 1992; Hinic et al., 2008). Moreover, some multiplex PCR assays (e.g., AMOS, Bruceladder) were established for further species differentiation (López-Goñi et al., 2008; Mayer-Scholl et al., 2010). However, none of the available molecular typing systems cover all currently known species and biovars of the genus Brucella. In microbiological routine testing, the identification of species and biovars is based on specific properties of the bacteria (e.g., $\mathrm{CO}_{2}$ requirement, $\mathrm{H}_{2} \mathrm{~S}$ production, urease activity, agglutination with monospecific $A, R$, and $M$ sera, growth on media with thionin or basic fuchsin, metabolization of different substrates) and in particular susceptibility to lytic Brucella reference phages (Al Dahouk et al., 2010).

Phages which infect and lyse Brucella strains are known for over half a century (Parnas et al., 1958; Brinley-Morgan et al., 1960; Jablonski, 1962). After some basic characterization, a typing set comprising five reference phages [Tb (Tbilisi), Fi (Firenze), Wb (Weybridge), Bk (Berkeley), R/C] was developed (Corbel, 1984). Some years later the typing set was complemented by phage Iz (Izatnagar; Joint FAO/WHO Expert Committee on Brucellosis, 1986) and since then has been used in many diagnostic laboratories worldwide. The same holds true for a set of Brucella reference strains serving as controls for lysotyping. The original typing set has also been modified by adding other phages, e.g., S708, Bk2, F1, F25, and Np, some of which are mutants of the reference phages (Moreira-Jacob, 1968; Corbel et al., 1988; Rigby et al., 1989; Hammerl et al., 2014). All brucellaphages described so far have a podoviral morphology and are closely related, demonstrated by restriction analysis and southern hybridization (Segondy et al., 1988; Rigby et al., 1989). They are considered as a single taxonomic species (Corbel and Thomas, 1976; Ackermann et al., 1981). As a consequence, some phages possess an almost identical host specificity (Morgan, 1963; Calderone and Pickett, 1965). For a better identification and discrimination of isolates, Brucella lysotyping is mostly carried out by spot assays using both a routine test dilution (RTD), which is the highest dilution of a phage suspension producing confluent lysis of a propagator strain, and a 10,000 $\times$ RTD phage suspension. The main drawback of this procedure is that it cannot clearly distinguish between strains, in which the phages propagate, and those which are merely killed by the so-called lysis from without effect caused by a collapse of the cell wall in response to an overwhelming number of adsorbed phage particles (Corbel, 1984). Similar to growth inhibition, lysis from without is rather unspecific and more difficult to interpret than single plaques that unequivocally illustrate a phage infection (Jones et al., 1968). Furthermore, host range variants of Brucella reference phages have been isolated, particularly after changing the propagator strain (Corbel et al., 1988). Hence, it is not surprising that even studies, in which the same reference phages were tested, may show inconsistent results (Morris et al., 1973). For that reason it is important to know the biological and genetic properties of the typing phages exactly. First DNA sequences of phage $\mathrm{Tb}$ were reported by Zhu et al. (2009). The analysis of whole genome sequences of diagnostic brucellaphages confirmed their close relationship, even though phage $\mathrm{Tb}$ deposited in two different institutes revealed some sequence deviations, mainly point mutations (Flores et al., 2012; Farlow et al., 2014; Tevdoradze et al., 2015). Indeed, besides two InDels $\sim 2.4$ and 0.4 $\mathrm{kb}$ in size, the genomes of the investigated phages notably differ by single nucleotide polymorphisms (SNPs). Many of them were found in a gene probably encoding a tail collar protein, which has been suggested to be a determinant of host specificity. The importance of individual amino acids of the tail collar protein for the host range of the phages, however, has not yet been investigated.

In this work, the host specificity and genomes of six VLA Brucella reference phages (designated $\mathrm{Tb}_{\mathrm{V}}, \mathrm{Fi}_{\mathrm{V}}, \mathrm{Wb}_{\mathrm{V}}, \mathrm{Bk}_{\mathrm{V}}$, $\mathrm{R} / \mathrm{C}_{\mathrm{V}}$, and $\mathrm{Iz}_{\mathrm{V}}$ herein) were analyzed in detail. The genome sequences of $\mathrm{Bk} 2_{\mathrm{V}}$ and $\mathrm{Iz}_{\mathrm{V}}$ will be presented for the first time. The phages were compared with homonymous phages originating from other laboratories. Bioinformatic analyses revealed DNA repeats within the phage genomes, which may be important for the acquisition, loss or duplication of DNA sequences. The host range determination of 22 non-reference phages disclosed some potential candidates useful for lysotyping.

\section{MATERIALS AND METHODS}

\section{Bacterial Strains, Media, and Growth Conditions}

Detailed information on all strains used in this study is given in Table S1. Cultivation of the bacteria was performed as previously described (Alton et al., 1975). Solid and overlay agar contained 1.8 and $0.7 \%$ (w/v) bacto-agar No. 1 (Oxoid, Wesel, Germany), respectively.

\section{Propagation of Bacteriophages}

Relevant data on brucellaphages used in this study are given in Table S2. The reference phages $\mathrm{Tb}_{\mathrm{V}}, \mathrm{Bk} 2_{\mathrm{V}}, \mathrm{W} b_{\mathrm{V}}, \mathrm{Fi}_{\mathrm{V}}, \mathrm{R} / \mathrm{C}_{\mathrm{V}}$, and $\mathrm{I}_{\mathrm{V}}$ were obtained from the Weybridge bacteriophage collection in form of unopened lyophilized phage stocks produced by the OIE Brucellosis Reference Centre of the Veterinary Laboratories 
Agency (VLA, Addlestone, UK) in 1973. Lyophilized phages were suspended in $5 \mathrm{ml}$ of SM-buffer $(100 \mathrm{mM} \mathrm{NaCl}, 8 \mathrm{mM}$ $\mathrm{MgSO}_{4} \quad 7 \mathrm{H}_{2} \mathrm{O}, 50 \mathrm{mM}$ Tris- $\mathrm{HCl}, \mathrm{pH}$ 7.5). The suspensions were used for plaque assays by the softagar overlay method as previously described (Sambrook and Russell, 2001). In contrast to phage $\mathrm{R} / \mathrm{C}_{V}$, which was propagted on $B$. ovis strain $63 / 290$, $\mathrm{Tb}_{\mathrm{V}}, \mathrm{Fi}_{\mathrm{V}}, \mathrm{Bk} 2_{\mathrm{V}}, \mathrm{Wb}_{\mathrm{V}}$, and $\mathrm{Iz}_{\mathrm{V}}$ were propagated on B. abortus vaccine strain $S 19$. Besides the standard reference phages which are globally used for Brucella-typing, other brucellaphages were included in the study. While lyophilized A422 and M51 stocks from 1960 were also provided by VLA, the origin of the phages F1, F1m, F1u, F25, F25u, F44, F45, F48, FO1, P, $\mathrm{P} 2,3,6,7,10 \mathrm{I}, 12 \mathrm{~m}, 24 \mathrm{II}, 45 \mathrm{II}, 212 \mathrm{XV}$, and 371XXIX is unknown. Lyophilized stocks of these phages prepared at the $\mathrm{BfR}$ in 1973 were used for further investigation. However, there is no information available on how these phages were propagated before lyophilization. To ensure the purity of all brucellaphages, a three-fold successive single plaque separation was performed. After the third plaque separation purified phages were used for the preparation of high titer lysates $\left(>10^{9} \mathrm{PFU} / \mathrm{ml}\right)$. To accomplish this, $10^{6} \mathrm{PFU}$ were applied to $20 \mathrm{ml}$ of an early logarithmic growing (McFarland 1.0-1.5) Brucella culture followed by incubation for $24-48 \mathrm{~h}$ on a rotational shaker (100 rpm) under microaerobic conditions. Thereafter, lysates were centrifuged for $10 \mathrm{~min}$ at 7,500 $\times \mathrm{g}$ to remove cellular debris. Supernatants were subjected to sterile filtration ( 0.45 and $0.2 \mu \mathrm{m}$; Merck Millipore, Schwalbach, Germany) and DNaseI/RNaseA-treatment (10 $\mu \mathrm{g} / \mathrm{ml}$ wt/vol each; Roche, Mannheim, Germany). Phage particles were concentrated and purified by discontinuous CsCl-step gradients $\left(\mathrm{CsCl}, 1.3\right.$ to $\left.1.7 \mathrm{~g} \mathrm{ml}^{-1}\right)$ as described previously (Sambrook and Russell, 2001). Phage bands recovered from CsCl-gradients were desalted using 100K Amicon Ultra centrifugal filter columns (Merck Millipore).

\section{Host Range Determination}

Host range analyses were carried out by spot assays on Brucella spp. reference and type strains $(n=26$; Table S1) and field isolates as well as reference strains of Ochrobactrum spp. $(n=119)$, Yersinia enterocolitica O:9 $(n=7)$, Mesorhizobium sp. $(n=6)$, Sinorhizobium meliloti $(n=5)$, and Pseudomonas ( $n=5$; data not shown). Two hundred microliters of each strain were mixed with $5 \mathrm{ml}$ of pre-warmed Brucella-broth soft agar $(0.7 \%)$ and poured onto a lysogeny-broth (LB) agar plate. Ten microliter aliquots of 1:10 serial dilutions of each lysate were spotted onto the overlay agar. Agar plates were visually inspected after incubation for 24 and $48 \mathrm{~h}$ at $37^{\circ} \mathrm{C}$. Phages that did not affect bacterial growth were classified as non-infectious (negative: - ). Phages were classified as infectious (positive: + ) when single plaques were identified in spotting zones of the tested lysates. In case of growth inhibition (GI) visible by an even but decreased bacterial growth within the spotting areas, the respective phage/strain combinations were further investigated by preparing phage lysates. For this purpose, $10^{7}$ phages were applied to $20 \mathrm{ml}$ of an early exponential growing Brucella culture (McFarland: $1.0-1.5$ ). After incubation at $37^{\circ} \mathrm{C}$ for $48 \mathrm{~h}$, lysates were purified as described above. Propagation of phages was determined by plaque assays (Sambrook and Russell, 2001). Four hundred microliters of a Brucella culture (McFarland: 5.0-7.0) were mixed with $100 \mu \mathrm{l}$ aliquots of 1:10 serial dilutions of each lysate, incubated for $20 \mathrm{~min}$ at room temperature, added to $5 \mathrm{ml}$ of pre-warmed Brucella-broth soft agar $(0.7 \%)$ and poured onto LB agar plates. After incubation for 24 and $48 \mathrm{~h}$ at $37^{\circ} \mathrm{C}$, agar plates were visually inspected for plaque activity. If phage replication occurred, the lysates contained more than $10^{7}$ infectious particles. Strains which increased the phage titer were finally classified as susceptible (positive: + ).

\section{Isolation of Phage DNA, Whole Genome Sequencing, and Bioinformatic Analysis}

Phage DNA extraction from CsCl-purified particles was performed as previously described (Hammerl et al., 2016). Determination of phage genomic sequences was conducted using a Roche 454 genome sequencer FLX titanium system by GATC Biotech AG (Konstanz, Germany). Library generation and 454 FLX sequencing were carried out according to the procedure of the manufacturer (Roche/454 Life Sciences, Branford, Connecticut, USA). Sequence reads were assembled using the Roche/454 Newbler software at default settings (454 Life Sciences Corporation, Software release 2.3 ) resulting in one contig with an average sequence coverage of $>100$ per consensus base. Sequence analysis and alignments were carried out using Accelrys Gene v2.5 (Accelrys Inc., San Diego, CA, USA). Bioinformatic analysis and genome annotation were performed as described previously (Hammerl et al., 2014, 2016).

\section{PCR Analysis}

PCR was performed in an Eppendorf Mastercycler ep Gradient (Eppendorf, Hamburg, Germany) according to standard protocols. Single reactions were carried out with $\sim 10 \mathrm{ng} / \mu \mathrm{l}$ phage DNA and $2.5 \mu \mathrm{l}$ of each primer in a final volume of $50 \mu \mathrm{l}$ using Qiagen DNA polymerase amplification components (Qiagen, Hilden, Germany). For PCR amplification the following parameters were used: initial template denaturation at $96^{\circ} \mathrm{C}$ for $120 \mathrm{~s}$ followed by 35 cycles including denaturation at $96^{\circ} \mathrm{C}$ for $15 \mathrm{~s}$, annealing at $55^{\circ} \mathrm{C}$ for $5 \mathrm{~min}$ and elongation for $210 \mathrm{~s}$ at $72^{\circ} \mathrm{C}$. A final elongation step at $72^{\circ} \mathrm{C}$ for 1 min was added. Purification of PCR products was performed by using the MSB spin PCRapace kit (Stratec, Birkenfeld, Germany). The nucleotide sequence of the PCR products was determined by Sanger sequencing (Eurofins Genomics, Ebersberg, Germany).

\section{Nucleotide Sequence Accession Number}

The complete nucleotide sequences of the brucellaphages were submitted to GenBank under the accession numbers HF569092 $\left(\mathrm{Wb}_{\mathrm{V}}\right)$, HF569091 (TbV), HF569089 (Fiv), HF569091 (Bk2 $)$, HF569090 (R/C $)$, and KY056619 (IzV).

\section{RESULTS AND DISCUSSION}

\section{Host Range Determination of the VLA Brucella Reference Phages}

A comprehensive study with 10 Brucella species represented by 26 reference and type strains was performed to elucidate in which strains the group I to group VI Brucella reference 
phages $\mathrm{Tb}_{\mathrm{V}}, \mathrm{Fi}_{\mathrm{V}}, \mathrm{Wb}_{\mathrm{V}}, \mathrm{Bk} 2_{\mathrm{V}}, \mathrm{R} / \mathrm{C}_{\mathrm{V}}$, and $\mathrm{Iz}_{\mathrm{V}}$ (Corbel, 1987) replicate and which strains are merely lysed by lysis from without at high MOIs. Lytic activity of the phages was quantified by plaque assays (titration) allowing an accurate determination of the hosts' susceptibility. If no plaque formation occurred and only growth inhibition was observed, we examined propagation of the phages using liquid cultures of the respective strains (see section Materials and Methods). The phages $\mathrm{Tb}_{\mathrm{V}}$ (group I) and $\mathrm{Fi}_{\mathrm{V}}$ (group II) showed almost identical lysis patterns (Table 1). They infected reference strains of the eight $B$. abortus biovars (bv1-7, 9), B. neotomae 5K33, B. microti CCM4915 and two strains of the recently identified species $B$. vulpis (Scholz et al., 2016). On the other hand, $B$. suis 1,330 (bv1) was only lysed by phage $\mathrm{Tb}_{\mathrm{V}}$, but not by $\mathrm{Fi}_{\mathrm{V}}$. Data that have been published on the susceptibility of $B$. suis 1,330 to phage Tbilisi are rather contradictory. While several articles reported that this strain was not lysed by the phage at RTD (Jones et al., 1968; Flores et al., 2012), other authors obtained plaques with both Tbilisi and Firenze (Rigby et al., 1989). Another deviation from published data concerns $B$. melitensis 16M (bv1), for which we and also others could not confirm infection by Tbilisi and Firenze, whereas Rigby et al. (1989) reported on plaque formation caused by these phages (Rigby et al., 1989). This raises the question about the reasons for these discrepancies? One possible explanation is that in our study and in the study of Rigby et al. (1989), phages were propagated on the B. abortus vaccine strain S19 (bv1), which makes host adaptation as a reason for different lysis patterns improbable. Furthermore, the slightly different methodologies applied to determine lysis patterns might not account for diverging results. It is more likely that the phages and/or indicator strains used in the two studies were actually not identical, perhaps due to mutations in genes important for phage infection. Of course, this presumption can only be confirmed by whole genome sequencing of the used strains and phages. For phage Tbilisi different variants $\left(\mathrm{Tb}_{\mathrm{M}}\right.$ and $\left.\mathrm{T} \mathrm{b}_{\mathrm{Y}}\right)$ have already been described (Foster et al., 2007; Flores et al., 2012). In addition, it has been demonstrated that the propagation of brucellaphages on different indicator strains caused changes in the phage genome, which might alter host specificity (Tevdoradze et al., 2015). However, lysis patterns of the phages have not yet been determined and compared. We therefore address this issue in detail.

Unlike $\mathrm{Tb}_{\mathrm{V}}$ and $\mathrm{Fi}_{\mathrm{V}}$, the phages $\mathrm{Wb}_{\mathrm{V}}$ (group III), $\mathrm{Bk} 2_{\mathrm{V}}$ (group IV), $\mathrm{R} / \mathrm{C}_{\mathrm{V}}$ (group $\mathrm{V}$ ), and $\mathrm{Iz}_{\mathrm{V}}$ (group $\mathrm{VI}$ ) revealed lysis patterns that correlated well with published data. The species $B$. ceti and B. inopinata were not infected by any phage. Five phages inhibited the growth of the $B$. ceti reference strain but replication of the phages did not occur. Though, a number of B. ceti strains isolated from cetaceans were lysed by Weybridge and Izatnagar in another study (Foster et al., 2007). In contrast, for B. inopinata which is currently represented only by its type strain BO1, no lytic phage has been found so far. Since this strain harbors an active prophage (Hammerl et al., 2016) that might affect the susceptibility to other phages, the importance of the prophage was analyzed in more detail (see next chapter). To avoid misinterpretation of phage typing results, plaque assays allowing a quantitative determination of the lytic activity are certainly better suited than spot assays using high titer lysates, since lysis from without effects are too variable to identify species unequivocally. We also studied infection of other species (Ochrobactrum spp., Mesorhizobium sp., Sinorhizobium meliloti, Yersinia enterocolitica O:9, Pseudomonas sp.) by the brucellaphages and detected growth inhibition of some strains (data not shown). As the zones of growth inhibition looked similar to halos obtained with Brucella, such results could be misleading.

\section{Propagation of the Phages on Alternative Strains Did Not Alter Lysis Patterns}

In this set of experiments the question should be answered whether lytic specificity of the phages may be affected by a change of propagator strains. For that reason $\mathrm{Tb}_{\mathrm{V}}, \mathrm{Wb}, \mathrm{R} / \mathrm{C}_{\mathrm{V}}$, and $\mathrm{IzV}_{\mathrm{V}}$ were co-cultivated with $B$. abortus $\mathrm{S} 19, B$. melitensis $16 \mathrm{M}$, B. suis 1,330 and $B$. ovis $63 / 290$. After overnight incubation, phages were isolated and used for the next co-cultivation with the respective strain. This procedure was repeated 20 times corresponding to $\sim 500$ generations. Thereafter, host ranges of the phages were examined by plaque assays testing all reference and type strains (Table S1). Following this procedure, no change of lysis patterns was detected. Furthermore, no adaptation of the phages to new hosts was observed. Phage $\mathrm{R} / \mathrm{C}_{\mathrm{V}}$ e.g., remained its specificity and infected exclusively rough strains. The data suggest that even though propagation of brucellaphages on different strains may cause genomic changes (Tevdoradze et al., 2015) this is not necessarily associated with an alteration of the host range. However, the number of phage particles released from individual strains can differ significantly (data not shown). Thus, lysates of the same phage exhibited different titers which may bias a result. To avoid diverging lysis patterns, we recommend to propagate diagnostic brucellaphages on the same indicator strain and to examine the phage genomes by sequencing if results are inconsistent.

Since many phages have been isolated from Brucella cultures, a lysogenic state termed pseudolysogeny has been suggested for these phages (Renoux and Suire, 1963). Lysogeny may influence the susceptibility of the bacteria to phages. We investigated $B$. abortus S19 colonies that had survived infection by phage $\mathrm{Tb}_{\mathrm{V}}$. The isolated colonies were passaged several times. While a release of phage particles after mitomycin $\mathrm{C}$ treatment (Hammerl et al., 2016) was not observed, $\mathrm{Tb}_{\mathrm{V}}$ was identified in initial cultures by PCR. In addition, electron microscopy revealed $\mathrm{Tb}_{\mathrm{V}}$ particles adsorbed to the cell wall (data not shown). Even after repeated cultivation, the phage was no longer detectable. The data indicate that strain B. abortus S19 may serve as carrier for $\mathrm{Tb}_{\mathrm{V}}$ but that there is obviously no integration of the phage genome into the bacterial chromosomes. We also did not observe any immunity of the $\mathrm{Tb}_{\mathrm{V}}$-carrying bacteria against superinfection by the same or other Brucella reference phages. Similar results were published by other authors (Morris et al., 1973; Corbel and Morris, 1975). To elucidate whether $\mathrm{Tb}_{\mathrm{V}}$-induced cell lysis may be affected by endogenous prophages providing immunity to superinfection, a S19 derivative (S19lys) containing the temperate phage BiPBO1 (Hammerl et al., 2016) was studied. $\mathrm{Tb}_{\mathrm{V}}$ lysed the lysogenic strain like the original strain without 
TABLE 1 | Host range determination of Brucella reference phages.

Brucella spp. (Strain)

Brucella phage group

B. abortus
B. abortus ( 19$)$
B. abortus bv1 (544)
B. abortus bv2 (86/8/59)
B. abortus bv3 (Tulya)
B. abortus bv4 (292)
B. abortus bv5 (B3196)
B. abortus bv6 (870)
B. abortus bv7 (63/75)
B. abortus bv9 (C68)

1
$w_{b}$

II

III $B k 2 v \quad$ IV

$\begin{array}{cc}+ & + \\ 2 \times 10^{9} & 2 \times 10^{9} \\ 7 \times 10^{7} & 4 \times 10^{7} \\ 1 \times 10^{8} & 1 \times 10^{8} \\ 1 \times 10^{8} & 6 \times 10^{6} \\ 2 \times 10^{8} & 6 \times 10^{6} \\ 1 \times 10^{8} & 1 \times 10^{7} \\ 6 \times 10^{7} & 6 \times 10^{7} \\ 4 \times 10^{7} & 1 \times 10^{6} \\ 8 \times 10^{7} & 2 \times 10^{7}\end{array}$

B. melitensis
B. melitensis bv1 (16M)
B. melitensis bv2 (63/9)
B. melitensis bv3 (Ether)
B. suis
B. suis bv1 (1330)
B. suis bv2 (Thomsen)
B. suis bv3 (686)
B. suis bv4 (40)
B. suis bv5 (513)

$8 \times 10^{7}$

\pm
$1 \times 10^{7}$
$\mathrm{NL}$
$3 \times 10^{7}$

$3 \times 10^{7}$

+
$2 \times 10^{9}$
$2 \times 10^{8}$
$1 \times 10^{9}$
$1 \times 10^{7}$
$1 \times 10^{7}$
$1 \times 10^{8}$
$1 \times 10^{7}$
$3 \times 10^{8}$
$3 \times 10^{7}$

IV
$\mathbf{T b}_{\mathbf{V}}$
$+\mathbf{1}$
$2 \times 10^{9}$
$2 \times 10^{8}$
$1 \times 10^{8}$
$2 \times 10^{8}$
$3 \times 10^{7}$
$1 \times 10^{7}$
$2 \times 10^{7}$
$7 \times 10^{7}$
$3 \times 10^{7}$

+
$1 \times 10^{8}$
$1 \times 10^{7}$
$6 \times 10^{7}$

$3 \times 10$

$\begin{array}{cccc} \pm & - & \pm & \pm \\ 1 \times 10^{9} & \mathrm{NL} & 2 \times 10^{8} & 5 \times 10^{7} \\ \mathrm{Gl} & \mathrm{NL} & \mathrm{Gl} & \mathrm{Gl} \\ 1 \times 10^{7} & \mathrm{NL} & 3 \times 10^{7} & \mathrm{NL} \\ 5 \times 10^{8} & \mathrm{NL} & 4 \times 10^{8} & \mathrm{NL} \\ 5 \times 10^{8} & \mathrm{NL} & 5 \times 10^{8} & \mathrm{NL}\end{array}$

-
$N L$
$N L$
$N L$

\begin{tabular}{|c|c|c|c|c|c|c|}
\hline B. neotomae (5K33) & $1 \times 10^{9}$ & $2 \times 10^{7}$ & $2 \times 10^{8}$ & $1 \times 10^{8}$ & NL & $1 \times 10^{8}$ \\
\hline B. canis & - & - & - & - & - & - \\
\hline B. ceti & - & - & - & - & - & - \\
\hline B. ceti (B1/94) & $\mathrm{Gl}$ & $\mathrm{Gl}$ & $\mathrm{Gl}$ & $\mathrm{Gl}$ & NL & $\mathrm{Gl}$ \\
\hline B. pinnipedialis & + & - & + & - & - & - \\
\hline B. pinnipedialis (B2/94) & $4 \times 10^{6}$ & NL & $2 \times 10^{6}$ & $\mathrm{NL}$ & NL & NL \\
\hline B. innopinata (BO1) & $\mathrm{NL}$ & NL & $\mathrm{NL}$ & $\mathrm{NL}$ & NL & $\mathrm{NL}$ \\
\hline B. vulpis & + & + & + & + & - & + \\
\hline B. vulpis (FH6OHL) & $2 \times 10^{9}$ & $3 \times 10^{7}$ & $1 \times 10^{9}$ & $2 \times 10^{8}$ & NL & $5 \times 10^{8}$ \\
\hline B. vulpis (FH965HL) & $1 \times 10^{9}$ & $2 \times 10^{8}$ & $1 \times 10^{9}$ & $4 \times 10^{8}$ & NL & $2 \times 10^{8}$ \\
\hline
\end{tabular}

+, Plaque formation, -, no plaque formation. bv, biovar; NL, no lysis; Gl, growth inhibition.

the prophage. Thus, BiPBO1 did not affect $\mathrm{Tb}_{\mathrm{V}}$ propagation. However, lysates prepared with strain S19lys contained both $\mathrm{Tb}_{\mathrm{V}}$ and BiPBO1 which could be easily identified by their different plaque morphologies. As the BiPBO1 prophage was induced by infection with $\mathrm{Tb}_{\mathrm{V}}$, lysogeny has to be taken into account when lysates of brucellaphages are prepared. Otherwise, 
incorrect results might be obtained when the phages are used for typing.

\section{HindIII Restriction Analysis Is Suited to Allocate Bk2v and Izv to Existing Phage Groups}

Previous studies on the Brucella reference phages Tbilisi, Weybridge, $\mathrm{Bk} 2$, and $\mathrm{R} / \mathrm{O}$ (an instable variant of phage $\mathrm{R} / \mathrm{C}$ ) showed that their genomes cannot be distinguished by restriction analysis using the endonucleases BamHI, EcoRI, and PvuII, because identical fragment patterns were obtained (Segondy et al., 1988). In contrast, phage Nepean (Np) revealed some differences, e.g., an additional $1.0 \mathrm{~kb}$ fragment in the HindIII digest (Rigby et al., 1989). The hitherto sequenced brucellaphage genomes mainly differed by two major InDels and can thus be assigned to two groups (Flores et al., 2012; Farlow et al., 2014; Tevdoradze et al., 2015). The presence or absence of these sequences should be traceable by use of suitable restriction enzymes. We analyzed HindIII restriction patterns of the VLA reference phages in detail to ascertain whether the yet not sequenced phages $\mathrm{Bk} 2 \mathrm{~V}$ and $\mathrm{Iz}_{\mathrm{V}}$ belong to one of the existing groups. As documented in Figure 1, phage $\mathrm{Tb}_{\mathrm{V}}$ and $\mathrm{Fi}_{\mathrm{V}}$ showed two additional restriction fragments, $5.0 \mathrm{~kb}$ and $2.8 \mathrm{~kb}$ in size, which were absent in $\mathrm{Wb}_{\mathrm{V}}$ and $\mathrm{R} / \mathrm{C}_{\mathrm{V}}$. These fragments comprise DNA sequences that are missing in the latter phages. The fragments were also absent in the $\mathrm{Bk} 2 \mathrm{~V}$ restriction digest. This suggests that $\mathrm{Bk} 2_{\mathrm{V}}$ may exhibit identical deletions as Weybridge and R/C. The two above mentioned restriction fragments of $\mathrm{Tb}_{\mathrm{V}}$ and $\mathrm{Fi}_{\mathrm{V}}$ were detected in $I z_{V}$ indicating that this phage resembles $T b_{V}$ and $\mathrm{Fi}_{\mathrm{V}}$ in this respect. In summary, restriction analysis using HindIII is a fast and easy method to determine whether a new phage contains deletions similar to already known brucellaphages.

\section{The VLA Brucella Reference Phages Are Not Identical to Same-Named Phages in Other Laboratories}

Host range analyses showed that the six VLA reference phages infected a distinct range of strains, even though some phages revealed almost identical lysis patterns. The only difference that was observed between $\mathrm{Tb}_{\mathrm{V}}$ and $\mathrm{Fi}_{\mathrm{V}}$ was B. suis strain 1,330 that was infected by $\mathrm{Tb}_{\mathrm{V}}$ but not by Fiv. Similarly, the host range of $\mathrm{Wb}_{\mathrm{V}}, \mathrm{Bk} 2_{\mathrm{V}}$ and $\mathrm{Iz}_{\mathrm{V}}$ only differed by one or two strains (Table 1). To allow a comparison between host specificity and genome variations, the phages were sequenced, two of them $\left(\mathrm{Bk} 2_{\mathrm{V}}\right.$ and $\left.\mathrm{Iz}_{\mathrm{V}}\right)$ for the first time. The remaining four phages (Tbilisi, Firenze, Weybridge, and R/C) have already been characterized in previous studies but none of the genomic sequences determined in this work was identical to those described by other authors. $\mathrm{Tb}_{\mathrm{V}}$ showed eight SNPs compared to phage $\mathrm{Tb}_{\mathrm{W}}$ deposited at the Félix d'Hérelle Reference Center for Bacterial Viruses, Université Laval, Canada (Farlow et al., 2014). Five SNPs are silent mutations, two others lying within ORF21 (neck protein) and ORF44 (hypothetical protein) caused an amino acid exchange and one (within ORF14) is an insertion

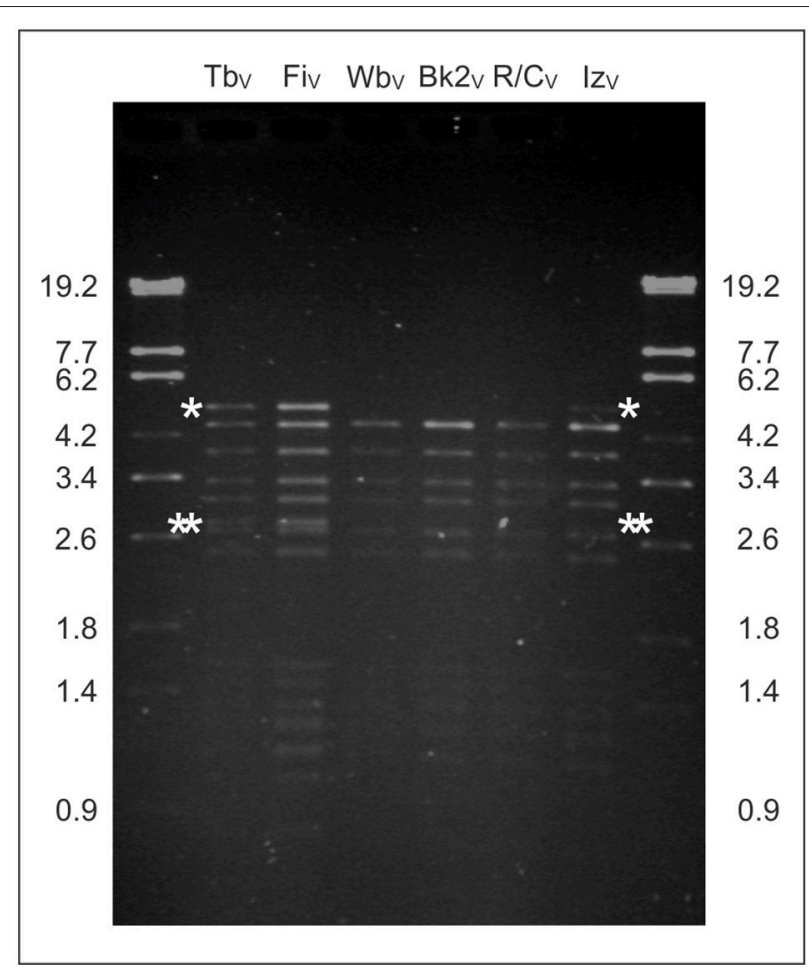

FIGURE 1 | HindIII restriction analysis of the VLA Brucella reference phages. Fragments that are present in $\mathrm{Tb}_{V}, \mathrm{Fi}_{V}$, and $\mathrm{Iz}_{\mathrm{V}}$ but absent in $\mathrm{Wb} \mathrm{b}_{V}$, $\mathrm{Bk} 2 \mathrm{~V}$, and $\mathrm{R} / \mathrm{C}_{\mathrm{V}}$ are marked by asterisks.

resulting in a truncated protein of unknown function. All these SNPs plus 14 additional SNPs exist when $\mathrm{Tb}_{\mathrm{V}}$ is compared with $\mathrm{Tb}_{\mathrm{E}}$ isolated at Eliava, Tbilisi, Ukraine (Tevdoradze et al., 2015). Differences to $\mathrm{Tb}_{\mathrm{M}}$ (Gamaleya Scientific Research Institute of Epidemiology and Microbiology, Moscow, Russia) are even more pronounced and have already been addressed by Farlow et al. (2014). Contrary to $\mathrm{Tb}_{\mathrm{V}}, \mathrm{Fi}_{\mathrm{V}}$ exhibited only two SNPs compared to Firenze in Laval. One SNP is located in an intergenic region, the other SNP caused an amino acid exchange at the $\mathrm{C}$-terminus of a hypothetical protein. $\mathrm{Wb}_{\mathrm{V}}$ showed five single nucleotide deviations to its counterpart in Laval. Three of them are located in genes (ORF16 and ORF23) for structural proteins, one in ORF27 probably encoding a tail collar protein and one in ORF57 for a primase/DNA polymerase. All of them caused amino acid exchanges. The most pronounced discrepancies between two phages with identical designations were found in $\mathrm{R} / \mathrm{C}$. The genomic sequence of the VLA R/C phage strain is 45 bp shorter than that of R/C in Laval. Furhermore, nine SNPs and two InDels were identified. Most deviations (four amino acid exchanges and one deletion of two amino acids) were found in the tail collar protein. Two frame shift mutations leading to radically changed gene products are present in ORF11 and ORF14 encoding a hypothetical protein and a primase/DNA polymerase, respectively. Quite the opposite was observed for $\mathrm{Bk} 2 \mathrm{~V}$. The host range of this phage differs significantly from that of Bk (Corbel, 1987; Farlow et al., 2014) but on the genome only one SNP located in the tail 
collar protein gene leading to an amino acid exchange was detected.

Phage $\mathrm{Iz}_{\mathrm{V}}$ exhibits the largest genome (41,446 bp) of all hitherto described brucellaphages. It contains a 301 bp duplicated nucleotide sequence located between the ORFs 23 and 24, which code for tail fiber proteins. Apart from this deviation, the $\mathrm{Iz}_{\mathrm{V}}$ genome composition is similar to those of $\mathrm{Tb}_{\mathrm{V}}$ and $\mathrm{Fi}_{\mathrm{V}}$ as it does not carry the two deletions present in other Brucella reference phages (Figure 2). However, based on the SNP data the closest relative of $\mathrm{Iz}_{\mathrm{V}}$ is not $\mathrm{Tb}_{\mathrm{V}}$ or $\mathrm{Fi}_{\mathrm{V}}$, but phage $\mathrm{Bk} 2_{\mathrm{V}}$. Besides the two InDels there are only five SNPs in these two phages. All of the SNPs are similarly present in the phages $\mathrm{Tb}_{\mathrm{V}}, \mathrm{Fi}_{\mathrm{V}}$, $\mathrm{Wb}_{\mathrm{V}}$, and $\mathrm{R} / \mathrm{C}_{\mathrm{V}}$. The SNPs are located in genes for the large terminase subunit, neck protein, a hypothetical protein and the primase/DNA polymerase (Table S3). $\mathrm{Tb}_{\mathrm{V}}$ and $\mathrm{Fi}_{\mathrm{V}}$ revealed 17 additional SNPs which are spread all over the phage genomes (Figure 2). Another interesting feature of $\mathrm{Iz}_{\mathrm{V}}$ is that the gene for the primase/DNA polymerase contains an internal stop codon, caused by the deletion of a single nucleotide. Therefore, in $\mathrm{Iz}_{\mathrm{V}}$ the largest gene of brucellaphages is splitted into two smaller ORFs. While the primase/DNA polymerase of $\mathrm{Tb}_{\mathrm{V}}$, for instance, comprises 780 amino acids, the ORFs 57 and 58 of $\mathrm{Iz}_{\mathrm{V}}$ encode polypeptides of 496 and 284 amino acids. Because of the frame shift mutation, the eight $\mathrm{C}$-terminal amino acids of the large $\mathrm{Iz}_{\mathrm{V}}$ polypeptide diverge from the $\mathrm{Tb}_{\mathrm{V}}$ protein. The small polypeptide exhibits no differences to the $\mathrm{Tb}_{\mathrm{V}}$ sequence. Our analysis of the $\mathrm{R} / \mathrm{C}_{\mathrm{V}}$ genomic sequence revealed that this phage also contains a stop codon within the primase/DNA polymerase gene. In this phage polypeptides of 236 and 555 amino acids are encoded. The data demonstrate that several variants of the primase/DNA polymerase exist in brucellaphages. Whether the two proteins of $\mathrm{Iz}_{\mathrm{V}}$ and $\mathrm{R} / \mathrm{C}_{\mathrm{V}}$ possess the same activity as their larger counterpart in $\mathrm{Tb}_{\mathrm{V}}$ is unknown and has to be clarified by further experiments.

On the basis of host range and whole genome analyses, Farlow et al. (2014) divided the Laval reference phages into three groups. Group I is composed of Tbilisi and Firenze, group II includes Berkeley, R/C and Pr from Mexico and group III contains Weybridge and S708. Some of these phages were also investigated in this study. However, since the VLA and Laval reference phages are not identical, they cannot be easily compared. From the data obtained in our study, the VLA reference phages can be allocated to four groups. As in the classification mentioned above, $\mathrm{Tb}_{\mathrm{V}}$ and $\mathrm{Fi}_{\mathrm{V}}$ belong to group I. Group II consists of $\mathrm{Wb}_{\mathrm{V}}$ and $\mathrm{Bk} \mathrm{V}_{\mathrm{V}}$, which exhibited an almost identical host range and which are genetically closely related. Phage $\mathrm{R} / \mathrm{C}_{\mathrm{V}}$ represents group III. It possesses an unique host specificity and showed some deletions and frame shift mutations not occurring in the other reference phages. The fourth group is represented by $\mathrm{Iz}_{\mathrm{V}}$ which does not fit to the other groups, neither by its host range, nor in terms of its genomic sequence. Due to the close overall DNA homologies of brucellaphages and because nucleotide variations can be observed after changing the host strain (Tevdoradze et al., 2015), the question arises, whether it really makes sense to group these phages. In addition, it should be considered that Brucella



FIGURE 2 | Genome organization of the VLA Brucella reference phages. The upper panel shows the gene map of IzV. Putative genes are colored according to the predicted functions of their gene products Table S4. SNPs identified by comparison with the other reference phages are indicated by orange rectangles (A-Q). For a better overview, only one SNP per gene is shown. A list with all SNPs is presented in Table S3. The lower panel illustrates the gene composition of the six VLA reference phages. Relevant genes are numbered. In W $b_{V}, B_{k} 2$ and R/C ORF27 and ORF28 are deleted, while ORF18 exhibits only are partial deletion. In Iz $\mathrm{C}_{V}$ and $\mathrm{R} / \mathrm{C}_{V}$ ORF57 is splitted into two ORFs IzV additionally contains the novel ORF 25 generated by sequence duplication. 
strains having the same designation do not need to be identical. This also can distort results, e.g., the determination of the host range of the phages.

\section{Identification of Repetitive DNA Sequences Possibly Involved in Genomic Rearrangements}

Sequence determination of Brucella reference phages disclosed a $2.4 \mathrm{~kb}$ DNA fragment comprising two genes for tail fiber proteins present in $\mathrm{Tb}_{\mathrm{V}}, \mathrm{Fi}_{\mathrm{V}}$, and $\mathrm{Iz}_{\mathrm{V}}$, but absent in $\mathrm{Wb}_{\mathrm{V}}, \mathrm{Bk}_{\mathrm{V}}$, and $\mathrm{R} / \mathrm{C}_{\mathrm{V}}$ (Figure 3). Subsequent InDel analysis revealed some remarkable consistencies among various phages. Compared to the other reference phages, $\mathrm{Wb}_{\mathrm{V}}, \mathrm{Bk} 2_{\mathrm{V}}$, and $\mathrm{R} / \mathrm{C}_{\mathrm{V}}$ show exactly the same deletion of $2,443 \mathrm{bp}$. In $\mathrm{Tb}_{\mathrm{V}}, \mathrm{Fi}_{\mathrm{V}}$, and $\mathrm{Iz}_{\mathrm{V}}$, the fragment is flanked by a 9 bp direct repeat termed RS-A and RS-B ( $5^{\prime}$ GACCAACCC- $3^{\prime}$, Figure 3). A third copy of this sequence (RSC) exists in reverse complement orientation $\sim 700$ bp apart from RS-A. By contrast, the $\mathrm{Wb}_{\mathrm{V}}, \mathrm{Bk} 2 \mathrm{~V}$, and $\mathrm{R} / \mathrm{C}_{\mathrm{V}}$ genomes contain only one copy of this sequence, adjacent to the deleted fragment. Notably, the $301 \mathrm{bp}$ sequence that is duplicated in $\mathrm{Iz}_{\mathrm{V}}$ also borders with one end on RS-A. At the other end of the duplicated sequence, a similar motif ( $5^{\prime}$-ACCAAACCC- $\left.3^{\prime}\right)$ is located in reverse complement orientation (Figure 3). This sequence does not exist in the other VLA Brucella reference phages. The duplication resulted in the generation of the new ORF 25 in $I_{V}$. These data suggest that the identified repeats may be important for the acquisition, loss or duplication of DNA sequences in brucellaphages. The additional $1.0 \mathrm{~kb}$ HindIII fragment identified in phage Nepean but not in other reference phages has also been suggested to be a repetition as it hybridized to Tbilisi DNA (Tevdoradze et al., 2015). It would be interesting to learn whether the repetition in this phage is similarly flanked by the repeats described above.

\section{New Phages May Be Helpful to Improve the Typing Set}

This study, and also those of other authors revealed several difficulties that may arise, when certain Brucella strains are typed using the existing reference phage typing set. The main reasons for this are the very similar host ranges of the phages and the fact that lysis from without effects, which are more difficult to interpret than plaques, have yet been included in the evaluation of lysis patterns. In addition, there are apparently host range variants of the reference phages, which further exacerbate the situation because it makes the comparison of data collected in different laboratories difficult. We therefore determined the host range of 22 non-reference phages deposited in the Brucella phage collection of the BfR (Table S2) to identify further candidates for typing. The analysis disclosed a very similar host specificity of the phages (Table 2). Though, the host range was clearly different from those of the reference phages. Like $\mathrm{Tb}_{\mathrm{V}}$ and $\mathrm{Fi}_{\mathrm{V}}$, none of the phages lysed strains of $B$. melitensis and $B$. pinnipedialis. All phages infected at least two, most of them even three $B$. suis biovars, namely bv1, bv4, and bv5. In this regard, the phages resemble $\mathrm{Wb}_{\mathrm{V}}, \mathrm{Bk} 2_{\mathrm{V}}$, and $\mathrm{Iz}_{\mathrm{V}}$. Lysis of Brucella species was similar to the reference phages. The exact analysis of the phages' host range also revealed some individual differences. This particularly pertained to the B. suis bv5 strain 513 that was not lysed by five phages. Brucella microti CCM $4915^{\mathrm{T}}$ was resistant only to one phage. Besides these specificities, all phages infected the same strains. It should, however, be emphasized that the phages produced a reduced number (up to 4 log units) of plaques on B. suis and B. microti strains. This has to be considered when the phages are used for typing. Two of the non-reference phages (A422 and M51) have been already studied by Morris et al. (1973). While we found plaques on B. suis with both phages, the other authors reported on lytic activity at RTD only for M51. It is conceivable that their A422 lysate did not contain enough active phage particles to cause lysis at RTD. Nevertheless, in spite of this discrepancy, the data of this study suggest that the reference phage typing set could be complemented by additional phages, which would make the discrimination of some Brucella species and strains more reliable.

The sequence analysis of the Brucella reference phages disclosed several SNPs (>50 SNPS, regions A-Q, Figure 2), most of them causing amino acid exchanges or deletions. Because no data were available about the genomes of the 22 non-reference phages, we examined all SNP positions by PCR using 23 different primer pairs. The study showed that all phages differed in at least one SNP and that none of the phages exhibited the two large deletions found in $\mathrm{Wb}_{\mathrm{V}}, \mathrm{Bk} 2_{\mathrm{V}}$, and $\mathrm{R} / \mathrm{C}_{\mathrm{V}}$. Taking into account all SNPs, two clusters of non-reference phages were assigned. One cluster (cluster B) consists of the phages A422 and M51 whose closest relatives are $\mathrm{Wb}, \mathrm{Bk} 2_{\mathrm{V}}, \mathrm{Iz}_{\mathrm{V}}$, and $\mathrm{R} / \mathrm{C}_{\mathrm{V}}$. The remaining phages form a cluster (cluster $\mathrm{C}$ ), which is related to cluster B (Figure 4). As the neck and tail collar protein genes of brucellaphages have been suspected to be important for host specificity (Flores et al., 2012; Farlow et al., 2014; Tevdoradze et al., 2015), we focused on the occurrence of SNPs within these genes. In the six VLA reference phages, the neck and tail collar protein genes exhibited five and seven SNPs, respectively. The analysis of the non-reference phages revealed even higher numbers of SNPs, some of which are located at the same position as in the reference phages (Table S3). A comparison of SNPs within the neck and tail collar protein genes with the host ranges of the phages did not provide evidence for amino acids that may decide on the strains that are infected. However, among 18 non-reference phages that exhibited an identical host range, 10 amino acid exchanges were observed in the neck protein and 11 in the tail collar protein, indicating that these positions do not determine specificity. Hence, even though the neck and tail collar protein genes are hotspots for nucleotide variations, it remains open which sequences are the key factor defining the host range of the phages.

\section{CONCLUSIONS}

Lytic phages have been applied for decades to identify and discriminate Brucella species and biovars. Moreover, the set of reference phages used in different laboratories is basically the same. The phages were isolated many years ago and they were distributed to diagnostic laboratories worldwide. The same holds true for Brucella reference and type strains serving as 


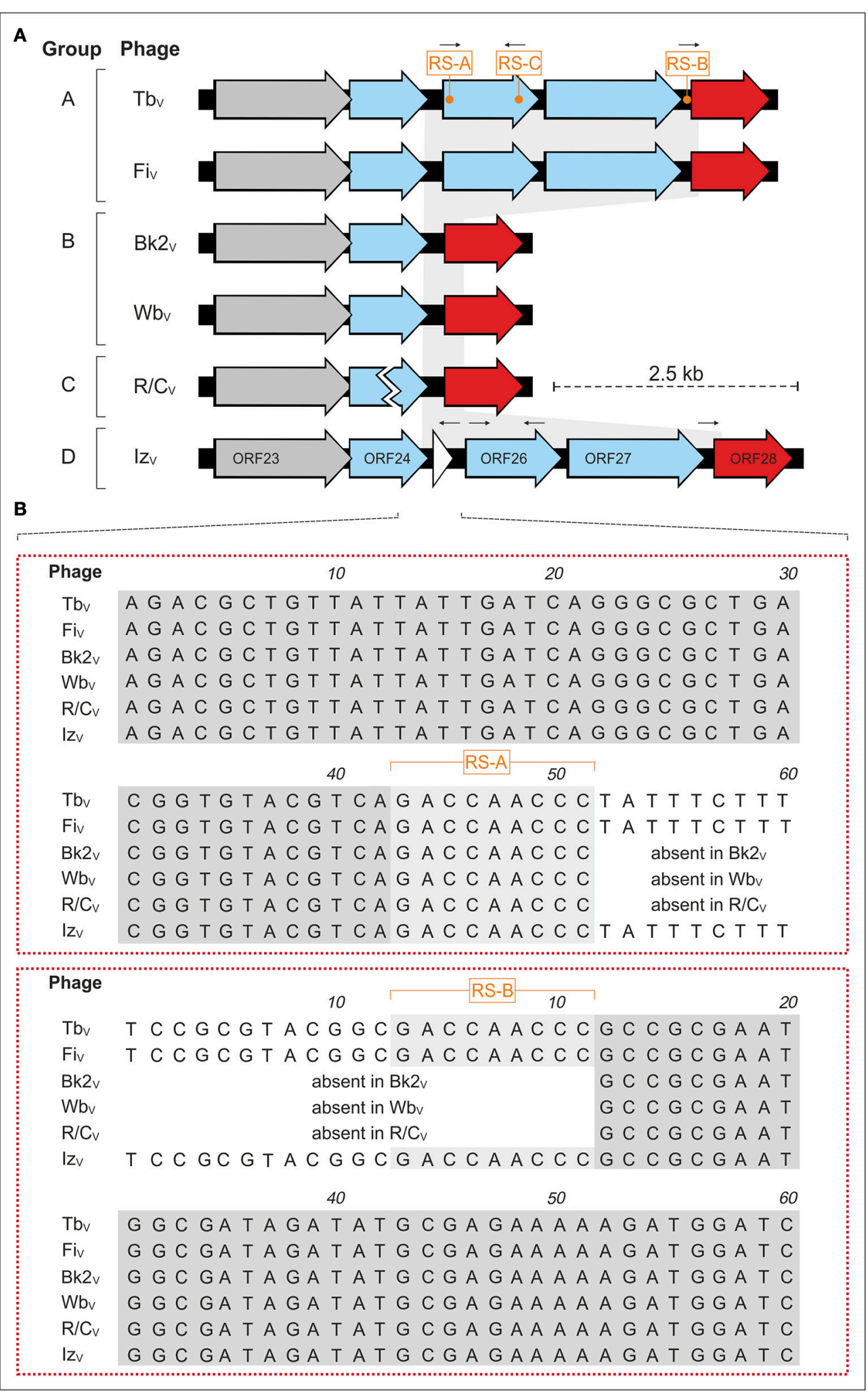

FIGURE 3 | Analysis of the large (2.443 bp) InDel in Brucella reference phages. (A) Gene arrangement in this DNA region. The position of the repeats RS-A, RS-B, and RS-C and of the additional inverted repeat present in Izv are indicated. (B) Alignment of the sequences flanking the InDel. The DNA fragment deleted in $\mathrm{Wb}_{\mathrm{V}}, \mathrm{Bk} 2 \mathrm{~V}$, and R/C $\mathrm{V}$ borders on the 9 bp direct repeats RS-A and RS-B. 
Hammer et al.

Genetic Diversity of Brucella Phages

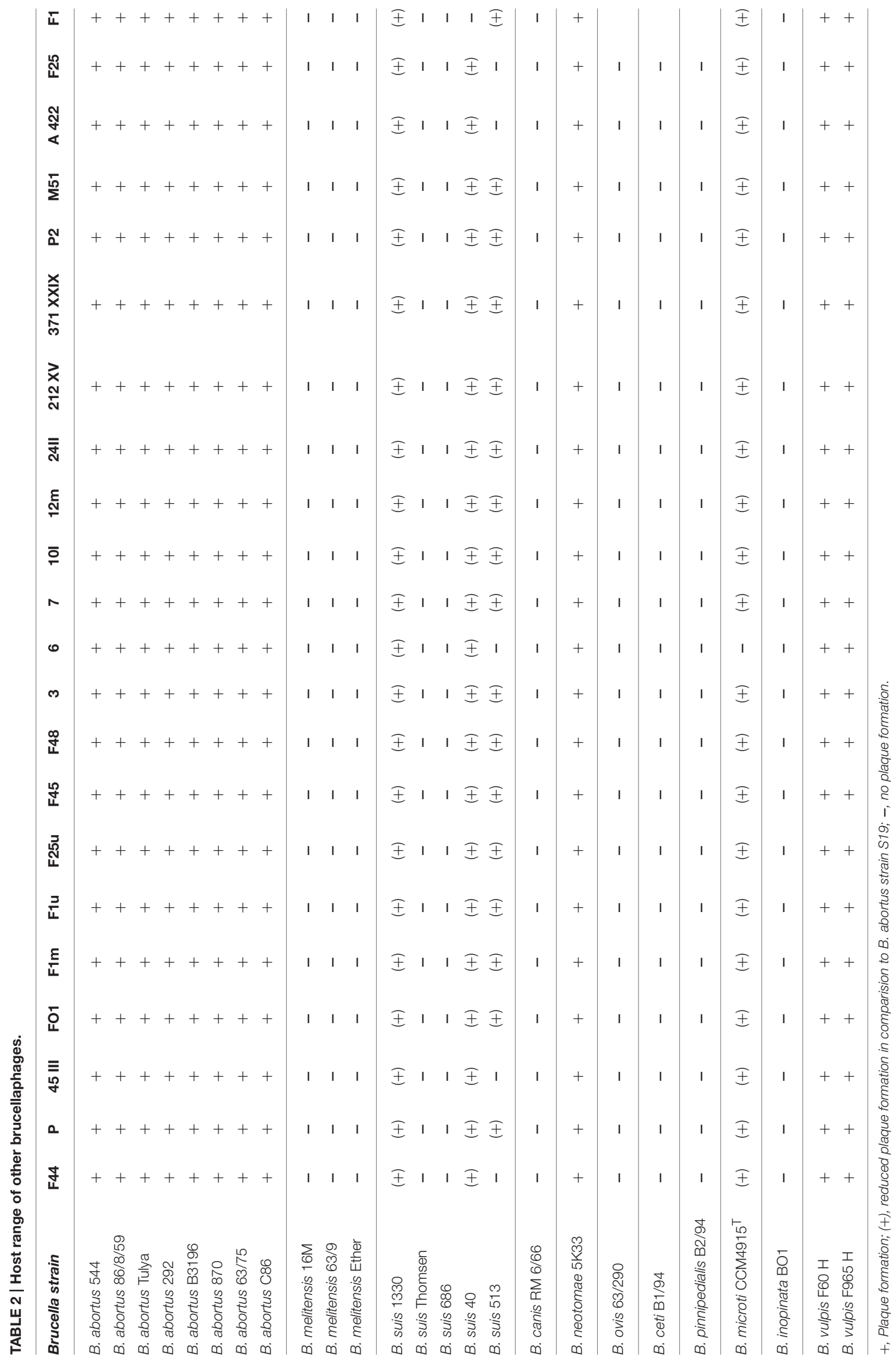

Frontiers in Microbiology | www.frontiersin.org

10

March 2017 | Volume 8 | Article 408 


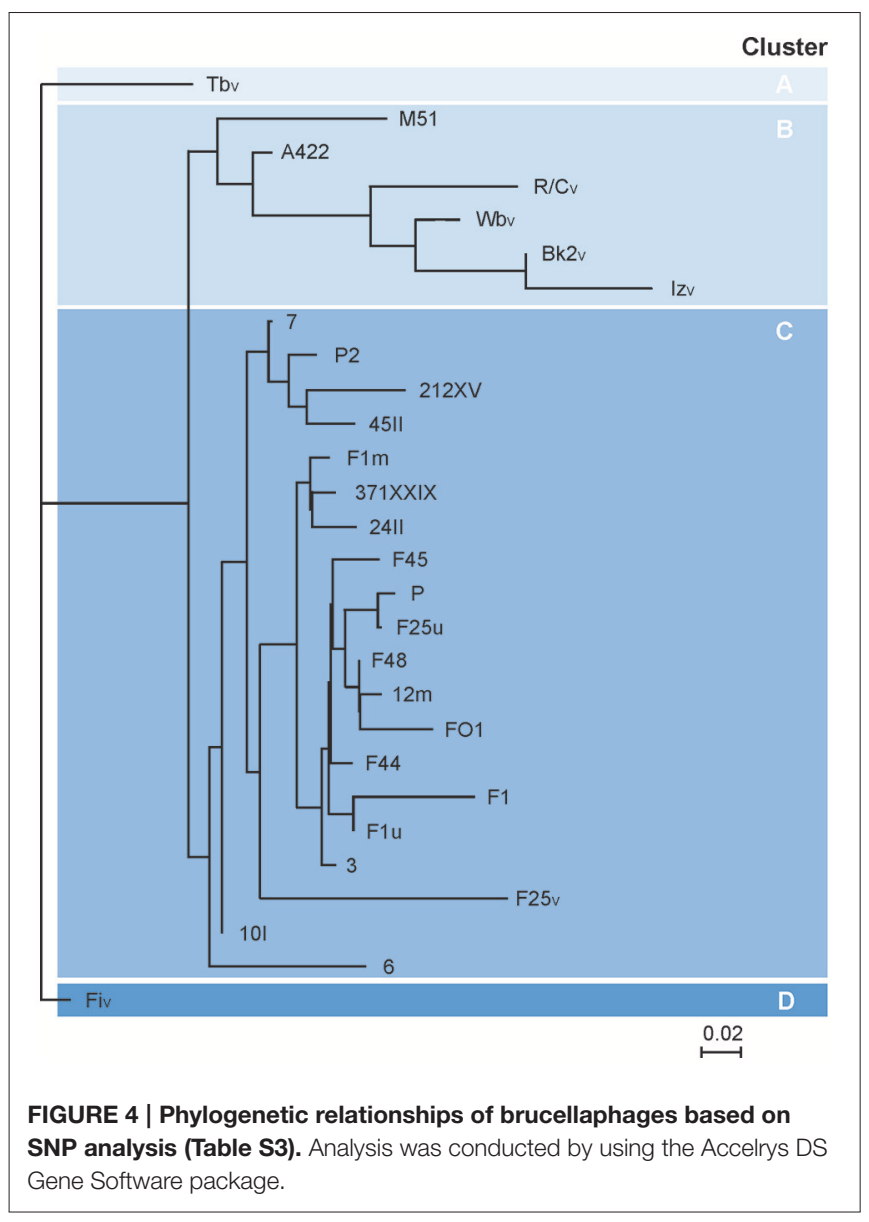

controls. After sequencing of the first Brucella reference phages, it became obvious that there is not only one Tbilisi, Firenze, or Weybridge phage but some variants of the prototypes revealing distinct sequence alterations. Nucleotide variations particularly occur in genes probably involved in host specificity. Therefore, it is not surprising that the VLA reference phages investigated in this study partially exhibited a host range different from homonymous phages in other laboratories. However, it is important to point out that in our study, lytic activity was defined as the ability of the phages to form plaques, while rather unspecific lysis from without effects caused by extremely

\section{REFERENCES}

Ackermann, H. W., Simon, F., and Verger, J. M. (1981). A survey of Brucella phages and morphology of new isolates. Intervirology 16, 1-7. doi: 10.1159/000149240

Al Dahouk, S., Scholz, H. C., Tomaso, H., Bahn, P., Göllner, C., Karges, W., et al. (2010). Differential phenotyping of Brucella species using a newly developed semi-automated metabolic system. BMC Microbiol. 10:269. doi: 10.1186/1471-2180-10-269

Alton, G. G., Jones, L. M., and Pietz, D. E. (1975). Laboratory Techniques in Brucellosis. Geneva: WHO

Baily, G. G., Krahn, J. B., Drasar, B. S., and Stoker, N. G. (1992). Detection of Brucella melitensis and Brucella abortus by DNA amplification. J. Trop. Med. Hyg. 95, 271-275. high numbers of phages were not evaluated. We think that this practice delivers more reliable data because single plaques are much easier to interpret than halos of lysis which may look very different. Results can also be biased when typing phages are propagated on different hosts, or when strains containing endogenous phages are infected. The B. abortus vaccine strain S19 is a well-suited host because it is susceptible to many Brucella phages and a Biosafety Level 2 (BSL2) organism. Thus, there are some issues that should be considered when brucellaphages are applied for typing. One main problem with lysotyping of Brucella strains is the similar host specificity of the reference phages. The situation could be improved by adding new phages to the typing set, which exhibit an individual host range. Our analysis of 22 non-reference brucellaphages revealed some new candidates that could be applied for routine diagnostics.

\section{AUTHOR CONTRIBUTIONS}

$\mathrm{JH}, \mathrm{HS}, \mathrm{KN}$, and $\mathrm{SH}$ designed the study. JH, CG, CJ, and JR performed the experiments. JH, CG, CJ, HS, JR, SA, and $\mathrm{SH}$ analyzed the data. $\mathrm{JH}, \mathrm{CJ}$, and $\mathrm{SH}$ wrote the manuscript and prepared the tables and figures. All authors edited the manuscript.

\section{ACKNOWLEDGMENTS}

The study was financially supported by a grant of the German Federal Institute for Risk Assessment (1332-488). Parts of the study were conducted within the Ess-B.A.R. project (FKZ: 13N13982).

\section{SUPPLEMENTARY MATERIAL}

The Supplementary Material for this article can be found online at: http://journal.frontiersin.org/article/10.3389/fmicb. 2017.00408/full\#supplementary-material

Table S1 | Bacterial strains used in this study.

Table S2 | Bacteriophages used in this study.

Table S3 | Single Nucleotide Polymorphisms (SNPs) among brucellaphages.

Table S4 | ORF analysis of brucellaphage Iz. 
Corbel, M. J., and Thomas, E. L. (1976). Properties of some new Brucella phage isolates; evidence for lysogeny within the genus. Dev. Biol. Stand. 31, 38-45.

Corbel, M. J., Tolari, F., and Yadava, V. K. (1988). Characterisation of a new phage lytic for both smooth and non-smooth Brucella species. Res. Vet. Sci. 44, 45-49.

de Figueiredo, P., Ficht, T. A., Rice-Ficht, A., Rossetti, C. A., and Adams, L. G. (2015). Pathogenesis and immunobiology of brucellosis: review of Brucella-host interactions. Am. J. Pathol. 185, 1505-1517. doi: 10.1016/j.ajpath.2015.03.003

Eisenberg, T., Hamann, H. P., Kaim, U., Schlez, K., Seeger, H., Schauerte, N., et al. (2012). Isolation of potentially novel Brucella spp. from frogs. Appl. Environ. Microbiol. 78, 3753-3755. doi: 10.1128/AEM.07509-11

Farlow, J., Filippov, A. A., Sergueev, K. V., Hang, J., Kotorashvili, A., and Nikolich, M. P. (2014). Comparative whole genome analysis of six diagnostic brucellaphages. Gene 541, 115-122. doi: 10.1016/j.gene.2014.01.018

Fischer, D., Lorenz, N., Heuser, W., Kämpfer, P., Scholz, H. C., and Lierz, M. (2012). Abscesses associated with a Brucella inopinata-like bacterium in a big-eyed tree frog (Leptopelis vermiculatus). J. Zoo Wildl. Med. 43, 625-628. doi: 10.1638/2011-0005R2.1

Flores, V., López-Merino, A., Mendoza-Hernandez, G., and Guarneros, G. (2012). Comparative genomic analysis of two brucellaphages of distant origins. Genomics 99, 233-240. doi: 10.1016/j.ygeno.2012.01.001

Foster, G., Osterman, B. S., Godfroid, J., Jacques, I., and Cloeckaert, A. (2007). Brucella ceti sp. nov. and Brucella pinnipedialis sp. nov. for Brucella strains with cetaceans and seals as their preferred hosts. Int. J. Syst. Evol. Microbiol. 57(Pt 11), 2688-2693. doi: 10.1099/ijs.0.65269-0

Godfroid, J., Al Dahouk, S., Pappas, G., Roth, F., Matope, G., Muma, J., et al. (2013). A "One Health" surveillance and control of brucellosis in developing countries: moving away from improvisation. Comp. Immunol. Microbiol. Infect. Dis. 36, 241-248. doi: 10.1016/j.cimid.2012.09.001

Hammerl, J. A., Al Dahouk, S., Nöckler, K., Göllner, C., Appel, B., and Hertwig, S. (2014). F1 and tbilisi are closely related brucellaphages exhibiting some distinct nucleotide variations which determine the host specificity. Genome Announc. 2:e1250-13. doi: 10.1128/genomeA.01250-13

Hammerl, J. A., Göllner, C., Al Dahouk, S., Nöckler, K., Reetz, J., and Hertwig, S. (2016). Analysis of the first temperate broad host range brucellaphage (BiPBO1) isolated from B. inopinata. Front Microbiol 7:24. doi: 10.3389/fmicb.2016.00024

Hinic, V., Brodard, I., Thomann, A., Cvetnic, Z., Makaya, P. V., Frey, J., et al. (2008). Novel identification and differentiation of Brucella melitensis, B. abortus, B. suis, B. ovis, B. canis, and B. neotomae suitable for both conventional and real-time PCR systems. J. Microbiol. Methods 75, 375-378. doi: 10.1016/j.mimet.2008.07.002

Jablonski, L. (1962). Variability of Brucella phages. Nature 193, 703-704. doi: $10.1038 / 193703 \mathrm{a} 0$

Jones, L. M., Merz, G. S., and Wilson, J. B. (1968). Phage typing reactions on Brucella species. Appl. Microbiol. 16, 1179-1190.

Joint FAO/WHO Expert Committee on Brucellosis (1986). Technical Report Series joint FAO/WHO Expert Committee on Brucellosis. World Health Organization.

López-Goñi, I., García-Yoldi, D., Marín, C. M., de Miguel, M. J., Muñoz, P. M., Blasco, J. M., et al. (2008). Evaluation of a multiplex PCR assay (Bruce-ladder) for molecular typing of all Brucella species, including the vaccine strains. J. Clin. Microbiol. 46, 3484-3487. doi: 10.1128/JCM.00837-08

Mayer-Scholl, A., Draeger, A., Göllner, C., Scholz, H. C., and Nöckler, K. (2010). Advancement of a multiplex PCR for the differentiation of all currently described Brucella species. J. Microbiol. Methods 80, 112-114. doi: 10.1016/j.mimet.2009.10.015

Moreira-Jacob, M. (1968). New group of virulent bacteriophages showing differential affinity for Brucella species. Nature 219, 752-753. doi: $10.1038 / 219752 \mathrm{a} 0$

Morgan, M. J. B. (1963). The examination of Brucella cultures for lysis by phage. J. Gen. Microbiol. 1963, 437-443. doi: 10.1099/00221287-30-3-437

Morris, J. A., Corbel, M. J., and Phillip, J. I. (1973). Characterization of three phages lytic for Brucella species. J. Gen. Virol. 20, 63-73. doi: 10.1099/0022-1317-20-1-63
Parnas, J., Feltynowski, A., and Bulikowski, W. (1958). Anti-Brucella phage. Nature 182, 1610-1611. doi: 10.1038/1821610a0

Renoux, G., and Suire, A. (1963). Spontaneous lysis and phage-carrier state in Brucella cultures. J. Bacteriol. 86, 642-647.

Rigby, C. E., Cerqueira-Campos, M. L., Kelly, H. A., and Surujballi, O. P. (1989). Properties and partial genetic characterization of Nepean phage and other lytic phages of Brucella species. Can. J. Vet. Res. 53, 319-325.

Sambrook, J. F., and Russell, D. W. (2001). Molecular Cloning: A Laboratory Manual, Vol. 3. New York, NY: Cold Spring Harbor Laboratory Press.

Scholz, H. C., Al Dahouk, S., Tomaso, H., Neubauer, H., Witte, A., Schloter, M., et al. (2008a). Genetic diversity and phylogenetic relationships of bacteria belonging to the Ochrobactrum-Brucella group by recA and 16S rRNA gene-based comparative sequence analysis. Syst. Appl. Microbiol. 31, 1-16. doi: 10.1016/j.syapm.2007.10.004

Scholz, H. C., Hubalek, Z., Sedlácek, I., Vergnaud, G., Tomaso, H., Al Dahouk, S., et al. (2008b). Brucella microti sp. nov., isolated from the common vole Microtus arvalis. Int. J. Syst. Evol. Microbiol. 58(Pt 2), 375-382. doi: 10.1099/ijs.0.65356-0

Scholz, H. C., Nöckler, K., Göllner, C., Bahn, P., Vergnaud, G., Tomaso, H. et al. (2010). Brucella inopinata sp. nov., isolated from a breast implant infection. Int. J. Syst. Evol. Microbiol. 60(Pt 4), 801-808. doi: 10.1099/ijs.0. 011148-0

Scholz, H. C., Pfeffer, M., Witte, A., Neubauer, H., Al Dahouk, S., Wernery, U., et al. (2008c). Specific detection and differentiation of Ochrobactrum anthropi, Ochrobactrum intermedium and Brucella spp. by a multi-primer PCR that targets the recA gene. J. Med. Microbiol. 57(Pt 1), 64-71. doi: 10.1099/jmm.0.47507-0

Scholz, H. C., Revilla-Fernández, S., Al Dahouk, S., Hammerl, J. A., Zygmunt, M. S., Cloeckaert, A., et al. (2016). Brucella vulpis sp. nov., isolated from mandibular lymph nodes of red foxes (Vulpes vulpes). Int. J. Syst. Evol. Microbiol. 66, 2090-2098. doi: 10.1099/ijsem.0.000998

Segondy, M., Allardet-Servent, A., Caravano, R., and Ramuz, M. (1988). Common physical map of four Brucella bacteriophage genomes. FEMS Microbiol. Lett. 56, 177-182. doi: 10.1111/j.1574-6968.1988.tb03173.x

Soler-Lloréns, P. F., Quance, C. R., Lawhon, S. D., Stuber, T. P., Edwards, J. F., Ficht, T. A., et al. (2016). A Brucella spp. isolate from a Pac-man frog (Ceratophrys ornata) reveals characteristics departing from classical brucellae. Front. Cell. Infect. Microbiol. 6:116. doi: 10.3389/fcimb.2016.00116

Tevdoradze, E., Farlow, J., Kotorashvili, A., Skhirtladze, N., Antadze, I., Gunia, S., et al. (2015). Whole genome sequence comparison of ten diagnostic brucellaphages propagated on two Brucella abortus hosts. Virol. J. 12:66. doi: 10.1186/s12985-015-0287-3

Whatmore, A. D., Dale, E. J., Stubberfield, E., Muchowski, J., Koylass, M., Dawson, C., et al. (2015). Isolation of Brucella from a White's tree frog (Litoria caerulea). JMM Case Rep. 2:e000017. doi: 10.1099/jmmcr.0.000017

Whatmore, A. M., Davison, N., Cloeckaert, A., Al Dahouk, S., Zygmunt, M. S., Brew, S. D., et al. (2014). Brucella papionis sp. nov., isolated from baboons (Papio spp.). Int. J. Syst. Evol. Microbiol. 64(Pt 12), 4120-4128. doi: 10.1099/ijs.0.065482-0

Zhu, C. Z., Xiong, H. Y., Han, J., Cui, B. Y., Piao, D., Li, Y. F., et al. (2009). Molecular characterization of $\mathrm{Tb}$, a new approach for an ancient brucellaphage. Int. J. Mol. Sci. 10, 2999-3011. doi: 10.3390/ijms10072999

Conflict of Interest Statement: The authors declare that the research was conducted in the absence of any commercial or financial relationships that could be construed as a potential conflict of interest.

Copyright (C) 2017 Hammerl, Göllner, Jäckel, Scholz, Nöckler, Reetz, Al Dahouk and Hertwig. This is an open-access article distributed under the terms of the Creative Commons Attribution License (CC BY). The use, distribution or reproduction in other forums is permitted, provided the original author(s) or licensor are credited and that the original publication in this journal is cited, in accordance with accepted academic practice. No use, distribution or reproduction is permitted which does not comply with these terms. 